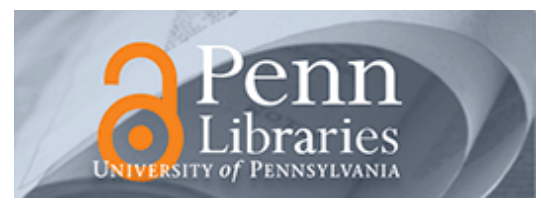

University of Pennsylvania

ScholarlyCommons

Marketing Papers

Wharton Faculty Research

2010

\title{
Creating Contagious: How Social Networks and Item Characteristics Combine to Drive Persistent Social Epidemics
}

Andrew T. Stephen

Jonah A. Berger

University of Pennsylvania

Follow this and additional works at: https://repository.upenn.edu/marketing_papers

Part of the Communication Technology and New Media Commons, Marketing Commons, Mass Communication Commons, Social Influence and Political Communication Commons, and the Social Media Commons

\section{Recommended Citation}

Stephen, A. T., \& Berger, J. A. (2010). Creating Contagious: How Social Networks and Item Characteristics Combine to Drive Persistent Social Epidemics. Retrieved from https://repository.upenn.edu/

marketing_papers/308

This is an unpublished manuscript.

This paper is posted at ScholarlyCommons. https://repository.upenn.edu/marketing_papers/308

For more information, please contact repository@pobox.upenn.edu. 


\title{
Creating Contagious: How Social Networks and Item Characteristics Combine to Drive Persistent Social Epidemics
}

\author{
Abstract \\ Why do certain cultural items capture persistent collective interest while others languish? This research \\ integrates psychological and sociological perspectives to provide deeper insight into social epidemics. \\ First, we develop a psychologically plausible individuallevel model of social transmission behavior. We \\ then situate this model in a social network and perform a series of simulations where we vary different \\ item- and networkrelated characteristics in an experimental setting. The results (1) demonstrate how item \\ and network characteristics combine to drive persistent collective enthusiasm and (2) shed light on the \\ underlying mechanisms through which such social epidemics occur. Interest in most items or products \\ naturally decays over time, so item characteristics (e.g., talkability) and the network positions of early \\ consumers are critical for bolstering consumer enthusiasm. Importantly, however, they do so via different \\ mechanisms, determining how frequently, and with what level of enthusiasm, items are discussed.

\section{Disciplines} \\ Business | Communication Technology and New Media | Marketing | Mass Communication | Social \\ Influence and Political Communication | Social Media

\section{Comments} \\ This is an unpublished manuscript.
}


Creating Contagious: How Social Networks and Item Characteristics Combine to Drive

Persistent Social Epidemics

\author{
ANDREW T. STEPHEN \\ JONAH BERGER
}


Andrew T. Stephen (Andrew.Stephen@insead.edu) is an Assistant Professor of Marketing at INSEAD, Boulevard de Constance, Fontainebleau 77305, France. Jonah Berger (jberger@wharton.upenn.edu) is an Assistant Professor of Marketing at The Wharton School, University of Pennsylvania, 700 Jon M. Huntsman Hall, 3730 Walnut Street, Philadelphia, PA 19104. The authors contributed equally to this work and are listed in reverse alphabetical order. The authors thank Don Lehmann, Christophe Van den Bulte, Duncan Watts, and seminar participants at Yahoo! Research for their helpful comments. 
Why do certain cultural items capture persistent collective interest while others languish? This research integrates psychological and sociological perspectives to provide deeper insight into social epidemics. First, we develop a psychologically plausible individuallevel model of social transmission behavior. We then situate this model in a social network and perform a series of simulations where we vary different item- and networkrelated characteristics in an experimental setting. The results (1) demonstrate how item and network characteristics combine to drive persistent collective enthusiasm and (2) shed light on the underlying mechanisms through which such social epidemics occur. Interest in most items or products naturally decays over time, so item characteristics (e.g., talkability) and the network positions of early consumers are critical for bolstering consumer enthusiasm. Importantly, however, they do so via different mechanisms, determining how frequently, and with what level of enthusiasm, items are discussed. 
Products, websites, and songs often capture collective interest. Some holiday gifts (e.g., Tickle-Me-Elmo) become the talk of the town, some new websites (e.g., Twitter, Foursquare, or Chatroulette) grab everyone's attention, and some television shows or movies generate lots of buzz. These are all examples of social epidemics, or instances where a sizable proportion of a population is interested in or enthusiastic about a particular cultural item, for at least a short period of time.

Despite their ubiquity, however, relatively little is known about these collective phenomena. How are some cultural items able to generate shared enthusiasm that is ongoing? Cultural items vary on different dimensions, people choose what to discuss, and these conversations are situated within a broader social network. But because enthusiasm decays over time (Moldovan, Goldenberg, and Chattopadhyay 2006; Wu and Huberman 2007), getting consumers to continue using and talking about any one item is challenging. In the absence of reinforcement, enthusiasm will decline. So how do item characteristics, transmission processes, and social networks combine to reinforce enthusiasm and thus shape persistent epidemics in collective interest?

This paper integrates psychological and sociological perspectives to deepen understanding of social epidemics. Building on existing literature, we build a dynamic and psychologically plausible micro-level model of how consumers transmit word-ofmouth (WOM) over social ties. We then situate consumers in a realistic social network structure and, using agent-based modeling, simulate hundreds of social epidemics where we manipulate aspects of the network (i.e., the position of the early consumers) and the items being shared (i.e., how likely they are to be discussed and how quickly enthusiasm for them declines) to examine how these factors shape persistent collective enthusiasm. 
The current research makes several contributions. First, past research has separately considered how either item (e.g., Berger and Milkman 2010; Heath, Bell, and Sternberg 2001) or network factors (e.g., Goldenberg, Libai, and Muller 2001; Libai, Muller, and Peres 2005; Watts 2002) shape collective outcomes, but in reality these aspects interact. By integrating these areas, we show how they can work in concert to reinforce consumers' enthusiasm and generate larger and more persistent social epidemics. Our results suggest that in addition to their independent contributions, item characteristics and social networks can have important compensatory effects, allowing epidemics to occur even in instances where they would not be predicted by each individual factor alone.

Second, research has examined product adoption (e.g., Bass 1969; Goldenberg, Libai, and Muller 2004; Golder and Tellis 2004; Rogers 1976), but there has been less attention to what leads collective enthusiasm to persist (e.g., ongoing consumption or continued WOM). Given consumer enthusiasm naturally decays over time (Moldovan et al. 2006), we examine how WOM from others can reinforce individual interest and both spread and maintain collective enthusiasm. This research is the first to show how various factors influence not only whether people do something once, but also whether their interest or enthusiasm persists.

Third, our findings shed light on the underlying process behind social epidemics. They highlight different mechanisms through which collective enthusiasm can be reinforced, such as influencing how often people talk about something, or how enthusiastically they talk about it. 
Finally, this work begins to investigate a relatively understudied area of consumer research. While diffusion and social contagion have received a great deal of attention in the marketing science literature (Godes and Mayzlin 2004, 2009; Goldenberg et al. 2001, 2004, 2009) much less is known about the underlying psychological and sociological processes of social transmission, word of mouth spread through social networks, and how these aspects combine to shape collective outcomes.

\section{PAST RESEARCH ON SOCIAL CONTAGION AND SOCIAL EPIDEMICS}

Collective dynamics, such as diffusion and cultural prominence, have been studied across numerous fields, including psychology, sociology, marketing, epidemiology, and economics. Past research tends to be siloed, however, taking either a macro approach (e.g., examining social network structure or predicting aggregate product adoption) or a micro approach (e.g., exploring psychological motivations for social transmission), but rarely considering both together.

\section{Macro Approaches}

Most work on social epidemics in marketing and sociology has focused on aggregate, macro-level outcomes. Researchers have built models to predict aggregate product adoption (Bass 1969; Goldenberg, Han, Lehmann, and Hong 2009; Toubia, Goldenberg, and Garcia 2010; Rogers 1976) or examine product lifecycles and new product innovation (e.g., Goldenberg et al. 2001; Golder and Tellis 1993). 
Other work has examined how social networks shape diffusion (e.g., Iyengar, Van den Bulte, and Valente 2010; Van den Bulte and Lilien 2001). Social networks map the ties between people and have various aggregate properties (e.g., density, or the proportion of dyadic ties that exist out of all possible ties among a set of people). These aggregate properties can have an important effect on epidemic size, or the number of people that ever adopt a product (Coleman, Katz, and Menzel 1957; Goldenberg et al. 2001). Large epidemics, for example, are more likely to occur in more densely interconnected networks (Watts 2002).

By focusing on aggregate-level structures (e.g., social networks) or outcomes (e.g., overall product adoption), however, work in this area has tended to ignore the individual-level micro transmission processes that drive these aggregate phenomena (though see Goldenberg et al 2001). The Bass (1969) model predicts aggregate adoption over time, for example, but says little about what drives these collective outcomes.

\section{Micro Approaches}

Psychologically oriented researchers have taken a more individual-level approach, focusing on how psychology shapes social transmission and cultural success. Some work for example, has considered how need for uniqueness or self-enhancement motives affect whether people share WOM (Cheema and Kaitaki 2010; Stephen and Lehmann 2010; Wojnicki and Godes 2008). Other work has gone beyond dyadic transmission to consider how item characteristics, or the fit between cultural items and human psychology, influence collective outcomes (Kashima 2008; Schaller, Conway, and Tanchuk 2002; Schaller and Crandall 2004). Building on natural selection, work on cultural selection 
suggests that the survival and propagation of culture depends on its ability to tap shared emotions or fit with peoples’ motivations or cognitive constraints. Stories vary in how disgusting or surprising they are, for example, and these characteristics may affect their success. People report greater willingness to share disgusting urban legends (Heath et al. 2001), and more surprising or awe-inspiring New York Times articles are more likely to be viral (Berger and Milkman 2010).

But while separate disciplinary traditions have individually considered social networks, or the link between item characteristics and psychological processes, there has been little attention to how these aspects might combine to shape collective outcomes (cf. Frenzen and Nakamoto 1993). Most sociologists have focused on aggregate, macro-level structures. But while network models are useful for identifying structural conditions under which epidemics are possible, little diffusion may actually occur if a particular item is unlikely to be transmitted. Conversely, most psychologists have focused on internal, micro-level processes. But while certain items may be highly viral, they will fail to spread widely if they start with people who are not well connected to the rest of the population. Truly understanding collective outcomes therefore requires comprehending how these different factors act in concert.

There has also been little attention to what leads interest to persist. Existing models typically focus on product adoption (e.g., Bass 1969; Goldenberg et al. 2001). Coleman et al.’s (1957) classic Medical Innovation study, for example, examined whether social contagion influenced whether doctors ever prescribed a new drug. But while adoption is useful, it only tells part of the story. Fifty people might try a website or new soft drink, but adoption does not distinguish whether they continue to be 
enthusiastic, or simply try it once (see Libai, Muller, and Peres 2009). Consequently, research is needed that moves beyond adoption to consider ongoing usage, enthusiasm, and interest (Libai et al. 2010).

\section{THE CURRENT RESEARCH}

To gain deeper insight into collective outcomes, we examine how item characteristics and social network structure combine to drive social epidemics. In particular, we focus on the interplay between (1) properties of items transmitted across social ties as well as (2) where initiators (Libai et al. 2005), or consumers who first learn about the item, are positioned in the network.

We focus on persistent epidemics in collective enthusiasm, or instances where a large number of consumers are excited about a particular cultural item. The item is talked about, people are interested in it, and it is part of the cultural zeitgeist.

Such complex, multi-faceted outcomes require a confluence of events. Rather than separate groups of consumers being enthusiastic at different points in time, many consumers must be simultaneously interested in a given item. Maintaining enthusiasm for a given item is particularly challenging given inherent desires for novelty (Hirschman 1980; Rogers and Shoemaker 1971) and competition among cultural items for consumers' limited time and interest (Mark 1998). While people may be enthusiastic about an item right after they hear about it, enthusiasm or interest often fades over time (Moldovan et al. 2006; Wu and Huberman 2007). Figure 1 illustrates how such natural decays in interest impacts enthusiasm spread and epidemic persistence (shading indicates 
level of enthusiasm, with black being 100\% enthusiastic). Enthusiasm is contagious and can be transferred between people through word of mouth. In the absence of decay (panel A) enthusiasm spreads rather quickly as people share their enthusiasm for the product with others. After only four time periods nine people already have some nonzero level of enthusiasm, and this will only continue to grow over time. In the presence of decay, however, diffusion is greatly muted (panel B; a relatively high level of decay). When enthusiasm is allowed to naturally decline from period to period, the contagion process dies quickly, resulting in no one with any enthusiasm after just four periods. Consequently, persistent social epidemics require that consumer enthusiasm be continually reinforced.

\section{[INSERT FIGURE 1 ABOUT HERE]}

We focus on how social transmission (e.g., WOM conversations) can reinforce enthusiasm and mitigate the negative impact of decay. If Maureen is really enthusiastic about a website, she will be more likely to talk to her friends about it, which should boost their enthusiasm for the site. How much their enthusiasm is impacted, however, will depend on how enthusiastically she talks about the website, or the size of the enthusiasm “dose” she transmits. Given that interest decays over time, consumers must receive enough doses of enthusiasm (e.g., have enough conversations with other enthusiastic people) of sufficient size, and within a finite period of time for their enthusiasm to remain persistently high. We explore both dose frequency (i.e., how often people are told about a given item) and dose strength/size (i.e., how much enthusiasm is transmitted in a conversation) as separable mechanisms for enthusiasm reinforcement. In particular, we consider how item and network characteristics combine to determine the frequency and 
strength of WOM transmission at the micro level, and thus impact epidemic size and persistence at the macro level.

To illustrate this process, consider consumers learning about a new website. Those who hear about it first (i.e., “initiators”) learn about it from an outside channel (e.g., as the "seeds" of a viral marketing campaign). This exogenous information makes them enthusiastic about the website, and their enthusiasm makes them more likely to tell others, which spreads their enthusiasm and gets others talking. This is analogous to forest fires: they start from a single spark or small fire that spreads, starting more fires that eventually can engulf an entire forest in flames (cf. Watts 2007).

But what if the initial spark or fire only has time to ignite a few nearby trees before it dies out? Given that enthusiasm naturally decays over time, early "fires” must be maintained if they are to continue to light other trees. Further, for the whole forest to burn at once (i.e., a large and persistent epidemic), the early fires must stay lit. This requires that later-generation fires help maintain the initial, early-generation flames that ignited them in the first place.

One way people can continually reinforce one another's enthusiasm is through word-of-mouth (WOM). If no one talks to the initiators, or the people that subsequently learned about the website, their enthusiasm will naturally decay and their fires will go out. This not only directly reduces the size of the overall epidemic, but also indirectly contributes to its demise because they no longer spread enthusiasm, which is essential for keeping the overall forest burning. Hence, enthusiasm that flows out from initiators also needs to cycle back to keep them (and those near them) enthusiastic. These cycles of enthusiasm reinforcement are critical for epidemic persistence. 
We suggest that characteristics of the item and the network combine to shape enthusiasm reinforcement. First, as discussed above, decay is an item characteristic that works against large, persistent epidemics. Some products are easier to stay excited about than others, meaning that enthusiasm for them naturally declines at a slower rate (i.e., fires tend to stay lit). Second, some things are intrinsically more interesting or more likely to be brought up in conversation (i.e., are more "talkable”). This increases the chance that people who know about it will talk about it, which should increase diffusion. Third, how far initiators are from others in the network should impact how easily information can spread. The more closely tied initiators are to others, the more easily enthusiasm can spread outward in a relatively quick manner such that doses of enthusiasm do not decay too much along the way. Decay and talkability are item characteristics, and initiator network position is a network characteristic.

These aspects also work in concert. A website may be inherently talkable (the trees are dry and hence flammable) but if the initiators are not closely tied to everyone else in the network (the initial spark is in a remote corner of the forest), decay will likely overwhelm any epidemic before it gets off the ground. Alternatively, the initiators might be very central and closely tied to others in the network (the initial spark is in the middle of the forest), but if the item itself is unlikely to be talked about (the trees are very damp and not very flammable), epidemics will also be unlikely. Overall then, item and network characteristics may combine to provide reinforcement that generates persistent epidemics by mitigating the negative effect of decay.

Given our interest in understanding how individual-level processes shape collective outcomes we use methods from complex systems research (agent-based 
modeling; see Goldenberg et al 2001; Goldenberg, Libai, Moldovan, and Muller 2007;

Libai et al 2005; Lusch and Tay 2004). This approach can generate important insights into complex, multiply determined social phenomena in situations where other types of data would be impossible to obtain. First, we develop a psychologically plausible individual-level model of social transmission. We then situate this model in a social network and run simulations where different item- and network-related characteristics are experimentally varied. Analyzing the resulting data provides insight into how item and network characteristics combine to drive epidemics.

\section{AN INDIVIDUAL-LEVEL MODEL OF SOCIAL TRANSMISSION BEHAVIOR}

This section describes our model of consumer enthusiasm, reinforcement, and WOM transmission (see Figure 2 for a summary). While it is difficult to incorporate everything in a parsimonious model, we have tried to capture the main aspects of the process while avoiding unnecessary complexity. See appendix A for technical details.

\section{[INSERT FIGURE 2 ABOUT HERE]}

\section{Individual Actions and Underlying Enthusiasm}

Consider consumer $i$, who belongs to a social network of $N$ consumers (described below). A new product or cultural item is introduced into this population at time $t=1$. Consumer $i$ has a certain level of enthusiasm for this item at each point in time which determines the actions she takes. The more enthusiastic she is, (1) the more likely she will be to talk about the item with one of her friends, and (2) the more enthusiastically 
she will do so, which will have a greater boost on her friend's enthusiasm (details of this process are described below). These interactions occur repeatedly over time, and, for convenience, we break time into discrete periods. In a single period consumer $i$ can transmit WOM to just one of her friends, so we track time as successive one-on-one social encounters. Formally, let $Y_{i t} \in[0,1]$ be person $i^{\text {'s }}$ enthusiasm for the focal cultural item at the beginning of period $t$. $Y_{i t}$ starts at 0 and remains there until person $i$ hears about the item from a friend and receives a dose of enthusiasm.

\section{Enthusiasm Dynamics and Decay}

As noted earlier, in the absence of intervention, enthusiasm or interest in a given cultural item tends to naturally decay over time (Moldovan et al. 2006; Wu and Huberman 2007). We capture this by assuming that consumer $i$ 's enthusiasm decreases between the end of one period and the start of the next, until it reaches zero. Let the beginning-of-period $t$ enthusiasm be $Y_{i t}=\left(1-\delta_{i}\right) \cdot Y_{i, t-1}^{*}$, where $Y_{i, t-1}^{*}$ is the enthusiasm at the end of the previous period, and the decay rate is $\delta_{i} \in[0,1]$. This rate is predominantly an item characteristic: some cultural items hold interest longer than others. Further, to account for some people having shorter attention spans than others there is also a consumer-specific component. Specifically, for a given item, individuals’ decay rates are distributed around an item-specific decay rate (see appendix A for details).

\section{Social Encounters and Deciding Whether or Not to Talk About the Focal Item}

Enthusiasm is reinforced when consumers receive WOM about the item from one of their network ties (i.e., friends). For simplicity, we focus on dyadic transmission (i.e., 
one-to-one), and only allow enthusiasm to be transmitted if a consumer talks about a particular item in a given period.

Each consumer talks to one of his or her social ties about something every period. Let consumer $j$ be a "transmitter" for the sake of the following illustration. Suppose that in each period consumer $j$ (for all $j=1, \ldots, N$ ) randomly selects one of her friends as a potential conversation partner; call her $i$ (i.e., consumer $j$ is the would-be WOM transmitter and $i$ is the would-be receiver; note that $i$ herself can have another encounter with either $j$ or a different friend in the same period in which $i$ would be the transmitter). Consumer $j$ (transmitter) talks with consumer $i$ (receiver) in this encounter, though they need not talk about the focal item. In reality they could talk about any number of things. To keep things simple, we look at whether transmitters talk about the focal item or not (i.e., anything else or an "outside item").

What people talk about is a highly complex, multiply determined stochastic social process and we attempt to capture some of the key elements in this model. Conditional on $i$ and $j$ talking, let $p_{i j t}$ be the probability that $j$ talks about the focal item to $i$ in period $t$. Whether that item ends up being the topic of conversation is based on $p_{i j t}$. We consider two components that affect $p_{i j t}$ : (1) an actor-specific component related to how enthusiastic the transmitter and receiver are about the focal item when they talk, and (2) an item-specific component related to how likely the item is to be discussed, independent of the conversation dyad's own levels of enthusiasm.

Actor-Specific Component. Conversations usually involve balancing the interests of both parties. Whether consumer $j$ decides to talk to consumer $i$ about one thing or another depends not only on how enthusiastic the transmitter is, but also on how 
enthusiastic they think the receiver will be. People like to be listened to when they share WOM (Stephen and Lehmann 2010) and most people want conversations to go well. Consequently, transmitters will be more likely to bring up things that they think receivers will be interested in, even if they themselves are not highly enthusiastic. This behavior is also consistent with conversational norms (Grice 1975); i.e., people try to make informative and relevant contributions to conversations.

Some integration of the transmitter's and the receiver's enthusiasm has to be made. The respective weights placed on these two levels of enthusiasm likely vary across consumers. Some consumers may weight their own interests more heavily while others give more weight to their conversation partner's interests. Consequently, we allow for a mixture of these cases assuming that people are heterogeneous in the weight they put on their own versus a friend's enthusiasm when choosing a topic of conversation. This is specified as a simple linear combination for transmitter $j$ and receiver $i$ in period $t$ :

$$
\text { actor }_{i j t}=\gamma_{j} Y_{j t}+\left(1-\gamma_{j}\right) Y_{i t}
$$

Where $\gamma_{j} \in[0,1]$ is the self-versus-other enthusiasm weight that is specific to the transmitter $(j)$. Those who are more self-focused (other-focused) will have a higher (lower) value for $\gamma_{j}$ (see appendix A for details).

Item-Specific Component. Aspects of cultural items themselves also affect whether they are discussed. More surprising news articles or disgusting urban legends are more likely to be shared (Berger and Milkman 2010; Heath et al. 2001). Similarly, even people who have little interest in golf should be more likely to talk about Tiger Woods because he is culturally prominent (Fast, Heath, and Wu 2009). Consequently, independent of the transmitter's and receiver's levels of enthusiasm (i.e., the actor- 
specific component), the item itself should also influence what people talk about. We refer to this inherent probability of being talked about as an item's talkability. This is parameterized as $v \in[0,1]$ and is the probability that a person would talk about the focal item independent of how enthusiastic they and their friends are about it at that time.

Combining Actor- and Item-specific components. Combining these components, the formal specification for $p_{i j t}$ is simply the weighted average of actor- and item-specific components, both of which are probabilities, using weight $\alpha_{j} \in[0,1]$ :

$$
p_{i j t}=\alpha_{j} \cdot \text { actor }_{i j t}+\left(1-\alpha_{j}\right) \cdot v
$$

The weight between actor and item components $\left(\alpha_{j}\right)$ varies across transmitters to allow for heterogeneity in their sensitivity to how much their conversation topic choices are driven by enthusiasm versus item talkability. Simply, some people may be more inclined to talk about whatever is highly talkable despite theirs or their friends' interests (lower $\alpha_{j}$ ), whereas other people care less about general talkability and focus on whatever they and their friends are enthusiastic about (higher $\alpha_{j}$ ). We introduce this heterogeneity because it more closely mirrors reality than making an assumption about how consumers weight these two components when transmitting WOM (see appendix A for details).

Equation 2 gives the transmission probability $p_{i j t}$. Whether $j$ actually talks about the focal item to $i$ in period $t$ is then a $\operatorname{Bernoulli}\left(p_{i j t}\right)$ random variable, with $1=$ talk about focal item and $0=$ not talk about focal item.

\section{Transmitting Enthusiasm via Word-of-Mouth}

Given that consumers talk about a particular item (i.e., consumer $j$ talks to consumer $i$ about the focal item in period $t$ ), we next consider the dose of enthusiasm that 
gets transferred. Building on threshold contagion models (e.g., Leskovec, Adamic, and Huberman 2007; Watts and Dodds 2007), transmitters give receivers some dose of enthusiasm whenever they talk about an item. Let $d_{i j t} \in[0,1]$ be the dose size for the transmission of enthusiasm from $j$ to $i$ in period $t$. (Obviously if no conversation about the focal item occurs $d_{i j t}=0$.) Prior work has either used a fixed dose size or drawn it randomly from a predetermined distribution (cf. Dodds and Watts 2004). This is somewhat unrealistic, because the effect that a transmitter talking about an item has on the receiver should depend on how enthusiastically the transmitter talks about it as well as whether the receiver is inclined to listen. Consequently, we take into account both the transmitter's and receiver's enthusiasm levels in determining the dose (just as we did to determine transmission probability).

Dose $d_{i j t}$ lies between the transmitter and receiver's respective enthusiasm levels as follows: $d_{i j t} \sim \operatorname{Uniform}(a, b)$, where $a=\min \left(Y_{i t}, Y_{j t}\right)$ and $b=\max \left(Y_{i t}, Y_{j t}\right)$. This allows for the fact that while a transmitter might try to transmit a dose commensurate with their enthusiasm, the receiver's likelihood of listening (and thus the amount of enthusiasm received) will depend in part on the receiver's current level of enthusiasm (i.e., as a proxy for their "receptivity" to the message). It also allows for the fact that if a transmitter's enthusiasm is lower than the receiver's, the dose could be greater than the transmitter's enthusiasm because of the receiver's greater state of excitement (e.g., consider the enthusiasm-boosting effect a mere mention of Miley Cyrus can have on a fan who is already enthusiastic about Miley).

Consumer $i$ 's end-of-period $t$ enthusiasm (before decay is applied) will then be: 


$$
Y_{i t}^{*}=\left\{\begin{array}{clrr}
0 & \text { if } & Y_{i t}+d_{i j t}=0 \\
Y_{i t}+d_{i j t} & \text { if } & 0<Y_{i t}+d_{i j t}<1 \\
1 & \text { if } & Y_{i t}+d_{i j t} \geq 0
\end{array}\right.
$$

Note that the truncation on end-of-period enthusiasm is to restrict enthusiasm to the $[0,1]$ interval as per our above specification of a consumer's enthusiasm in this model.

\section{ENTHUSIASM TRANSMISSION OVER A SOCIAL NETWORK}

As noted previously, we are interested in how item- and network-related factors combine to drive social epidemics. The prior section described the individual-level dynamic model of enthusiasm for cultural items, its natural decay over time, and how social interactions and WOM can reinforce enthusiasm. Along the way, it discussed the two item characteristics we focus on, namely item talkability $(v)$ and decay $(\delta)$. We now turn to the network aspect, and apply this model to a socially networked population of $N$ consumers. We discuss how the network position of the initiators (i.e., how closely they are tied to others in the network) may shape persistent social epidemics

\section{Starting the Contagion Process Over the Social Network}

Certain Individuals Start the Process. As mentioned above, there is a set of $N_{\text {initiators }}<N$ consumers (with $N_{\text {initiators }}>0$ ) who we call "initiators." They start the contagion process that spreads WOM and enthusiasm about the item (e.g., they are the sparks that start the forest fire). They are the first to have heard about the item (e.g., through viral marketing campaigns or advertising), and at $t=1$ (i.e., when the focal item is introduced to the network) the initiators are enthusiastic about it. We assume that their 
enthusiasm level is above an exogenous, item-specific threshold, $\phi \in[0,1]$, consistent with threshold models used in extant literature (e.g., Castellano, Fortunato, and Loreto 2009; Dodds and Watts 2004; Watts and Dodds 2007). This threshold, although not of primary importance here, captures how easy (lower $\phi$ ) or hard (higher $\phi$ ) it is to be highly enthusiastic (or excited) about the focal item (see appendix B for details).

Selecting Initiators. We focus on where initiators are positioned in the social network, which is something that can be controlled by marketers or policy makers given some knowledge of the network's structure. We use a realistic structure that mimics social networks found in various natural settings. We do not consider variation in aggregate network characteristics (e.g., Watts 2002) because aggregate structures cannot be easily manipulated in reality.

In particular, consistent with our earlier discussion about how closely tied initiators are to everyone else, we focus on their centrality in the network. Degree, or the number of direct social ties (e.g., friends) people have, is the most commonly used centrality metric in extant research (e.g., Goldenberg et al. 2009). But while people with high degree may be good at getting the word out, they may be less useful when enthusiasm must be reinforced on an ongoing basis. Prior degree-based work has focused on one-time product adoption; i.e., where something needs to be transmitted only once between consumers. To reinforce enthusiasm in the face of decay, however, WOM needs to be transmitted repeatedly over time, and enthusiasm must repeatedly flow back-andforth between consumers. Consequently, initiators must not only start the contagion, but also play a role in keeping it alive, continuing to pump enthusiasm throughout the network. In other words, to spur one-time product adoption, enthusiasm only has to flow 
outward once (e.g., from A to B, B to C, and so on). But when reinforcement is needed, enthusiasm must also cycle back (to the initiators and everyone else along the path, e.g., from C to B and B to A). For initiators to play a role in keeping enthusiasm alive their own enthusiasm needs to be reinforced (as we noted in the forest fire example above).

This suggests considering the network distance between individuals instead of simply the number of connections that they have. The more intervening people between two indirectly connected consumers, the longer it will take a unit of enthusiasm to travel and the more enthusiasm that will be lost (since decay is time-dependent). Shorter paths, however, mean that enthusiasm spreads faster and less is lost to decay. In the case of enthusiasm flowing back-and-forth between initiators and everyone else, shorter cycles (i.e., path lengths out from initiators and back again) should thus be beneficial.

Consequently, we focus on closeness centrality as a criterion for initiator selection. Rather than first order connectivity (i.e., degree), closeness centrality (cf. de Nooy, Mrvar, and Batagelj 2005; see appendix C) focuses on average path lengths, or how closely tied an initiator is to everyone else (on average). This metric is higher (lower) for people who have a shorter (longer) average path length or "degrees of separation” between themselves and everyone else in the network. "Close” initiators (high closeness centrality) should be better for reinforcing enthusiasm than "peripheral" initiators (low closeness centrality) because there are fewer steps between them and the rest of the network. Further, when considering epidemic persistence, closeness should matter more than connectedness. Someone may have many friends (high connectedness), for example, but if they are on the outskirts of the network (low closeness) they are far 
from almost everyone else. Closeness takes into account distances between indirectly connected consumers, which should matter for reinforcing enthusiasm.

Figure 3 illustrates the impact of close and peripheral initiators in reinforcing enthusiasm flows. When a close initiator is used (panel A), she (node 1) initially spreads doses of enthusiasm (periods 1 and 2) and then later receives them back (periods 3 and 4), which reinforces her own enthusiasm. By virtue of her position in the network—only a short distance from many others - a close initiator is more likely to recoup larger doses of enthusiasm fairly quickly, before decay has had too much of a negative effect. In this case, even after four periods, the initiator has regained a high level of enthusiasm. This high level of enthusiasm increases the chance that the initiator will continue to transmit doses in the following periods, thus keeping the contagion process alive and spreading enthusiasm throughout the network.

Things go quite differently, however, when peripheral initiators are used (panel B). The initiator's position in the network makes it difficult for her (node 1) to reach out to many others in the first place to spread her enthusiasm, which then limits the potential for others to transmit reinforcing doses to her. Also, her distance from most others means that decay has a bigger effect in reducing enthusiasm cycles. The enthusiasm she shares is more greatly reduced before it flows back to her again, resulting in smaller doses and less overall enthusiasm. This leads contagion to die out soon after the fourth period.

[INSERT FIGURE 3 ABOUT HERE] 


\section{A STUDY OF HOW ITEM CHARACTERISTICS AND SOCIAL NETWORKS COMBINE TO DRIVE EPIDEMIC PERSISTENCE}

To investigate our questions of interest, we need (1) individual-level data on (2) ongoing enthusiasm as well as (3) WOM transmissions, for (4) multiple cultural items over (5) time in (6) a fully mapped social network. Since we are explicitly interested in how network and product characteristics interact, (7) initiators' network positions and (8) items’ characteristics need to vary across items.

Things are further complicated by the fact that empirical network research often suffer from endogeneity problems, making it hard to draw unbiased causal conclusions. A person's position in the network, for example, is likely correlated with unobserved person-specific characteristics that also influence her impact on the others around her. Close individuals are likely more personable, for example, and thus it may be their personality, rather than their network position that is driving any effect they have on epidemic persistence. Similarly, network structure is not constant or exogenous, and may change based on past social interactions. As a result, any observed diffusion could be driven by changes in structure rather than characteristics of the items themselves. Empirical network analyses also often suffer from omitted variable problems. Van den Bulte and Lilien (2001), for example, found that social contagion effects in the classic Medical Innovations data (Coleman et al. 1957) went away after advertising effects of were incorporated into the statistical model. Taken together, these various issues make it hard to use diffusion data to draw unbiased causal inferences about the effects of the network and item characteristics (and their interactions) on epidemic outcomes. 
To get around these issues, we turn to agent- based modeling. This approach allows us to manipulate various factors rather than just measure them, and study how the interaction between multiple micro-level factors in a complex system impacts macro outcomes. Further, it allows us to circumvent typical endogeneity problems inherent in empirical networks research. We can exogenously manipulate the key variables in our model and observe how they affect contagion, much like a controlled experiment. Moreover, we can exogenously control the environment in which the contagion process of interest is studied (i.e., structure of the social network over which enthusiasm spreads), which allows us to focus on only those factors of interest without concern for unintended effects of extraneous or unobserved factors on the outcomes.

Accordingly, we treat this simulation study as a laboratory experiment. We manipulate item characteristics (decay $\delta$ and talkability $v$ ) and the network position of the initiators (high vs. low closeness centrality) in a large full factorial design. (Note that we

also manipulate the enthusiasm threshold ( ) to show that the effects of the parameters of interest are robust to levels of this theoretically unimportant parameter.)

\section{Simulation Setup and Treatments}

We used a static, realistic network structure containing $N=1,000$ consumers (see appendix $\mathrm{D}$ for details). In each run of the simulation an item is introduced at $t=1$ by seeding a set of $N_{\text {initiators }}=20$ initiators ( $2 \%$ of the population). Each simulation runs for 200 periods, which we determined through pretests to be a sufficient length of time for stable outcomes to be reached (results are robust to shorter and longer runs). 
Table 1 lists the manipulated parameters and their levels. For the initiators, the 20 consumers with highest (lowest) closeness centrality were chosen in the "close" ("peripheral") condition. The item and initiator network position characteristics form a set of four factors that we manipulated in a 9 (decay) $\times 11$ (talkability) $\times 2$ (initiator position) $\times 4$ (threshold) full factorial design. This gives 792 cells.

\section{[INSERT TABLE 1 ABOUT HERE]}

\section{Data and Statistical Analysis}

For each simulation run (indexed by $k$ ), we tracked the time series of our main outcome variables: Talk $k$ and Excited $_{k t}$. For the $k^{\text {th }}$ run, Talk $k_{k t}$ tracks the item's "conversation share" as the proportion of the consumers who talked about the item (as opposed to something else) in period $t$. Excited ${ }_{k t}$ tracks the proportion of consumers who were excited about the item at the end of period $t$. This is a measure of collective enthusiasm for the item, and a consumer is deemed to be excited when their enthusiasm is high enough such that it exceeds the threshold $\phi$. An epidemic in collective enthusiasm for an item occurs when a large proportion of consumers in the population are excited. Thus, Excited $_{k t}$ is our main dependent measure of social epidemic size. For example, if the cultural item is a new song by Lady Gaga, Talk $k_{k t}$ is the proportion of consumers who spread WOM about the song in period $t$, and Excited $_{k t}$ is the proportion of consumers who were highly enthusiastic—excited—about the song at the end of period $t$.

Our simulations generated a panel dataset with $k=1, \ldots, 792$ items, each with $t=$ $1, \ldots, 200$ time-ordered observations. For each outcome variable, a dynamic random effects regression with cell random effects was estimated (Greene 2003). We regressed 
each outcome variable on a first-order autoregressive lag of itself (higher-order autoregressive lags were checked but did not improve fit), the excited threshold (as a control variable), and main effects, two-way interactions, and three-way interactions for decay, talkability, and initiator position. For Talk knt $_{\text {axcited }}$ ant the model was a Beta regression because these variables were proportions (see appendix E for details).

Our analysis is akin to regressing an outcome (e.g., Excited Ext $_{\text {) }}$ on a series of regressors that account for item characteristics (decay and talkability), the network position of initiators, and control variables (threshold, random effects for the specific simulation run, and the previous-period value [autoregressive lag] of the dependent variable). This last control—-the lagged dependent variable-is particularly important because it is used to determine the persistence of the effects of the item and network factors of interest. A significant positive effect of a lagged dependent variable indicates persistence of the effect over time.

\section{RESULTS}

\section{Manipulation Checks and Main Effects}

Parameter estimates and standard errors for the regression models for Talk $k_{k t}$ and Excited $_{k t}$ are reported in Table 2. Results show that the decay and talkability parameters operate as intended. While decay hurts conversation share and collective enthusiasm ( $p$ s $<.001)$, talkability has a positive main effect on both outcomes $(p s<.05)$. There were no main effects of initiator position ( $p$ s $>.40$ ), which was expected since the initiators’ positions are exogenous to the micro social transmission process model (whereas decay 
and talkability are parameters within the model itself). As we show below, initiator position plays a role through interactions.

[INSERT TABLE 2 ABOUT HERE]

Other effects that indicate the model operated as intended are: (1) the threshold $(\phi)$ has a significant negative effect on $\operatorname{Excited}_{k t}(p<.001)$ but not Talk $k$. Having a large proportion of consumers above the threshold is harder as the threshold increases, but consumers are free to spread WOM about the item even if their enthusiasm level is low. (2) The first-order autoregressive lag effects (each variable on itself) are positive and significant for each dependent variable ( $p$ s $<.001$ ), which, as mentioned above, indicates persistence. And (3) there is a significant positive effect of $\operatorname{Talk}_{k t}$ on $\operatorname{Excited}_{k t}(p<.001)$, which was expected because WOM increases enthusiasm and is directly linked to Excited $_{k t .}$ Finally, all models had good fit (pseudo- $\left.R^{2} \mathrm{~s}>.95\right)$.

\section{Interaction Effects: Mitigating Decay}

The analysis focuses on factors that can mitigate the negative effects of decay on

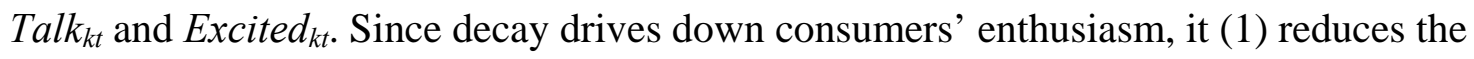
likelihood that the item is talked about, (2) reduces dose size, and as a result (3) makes social epidemics less likely to flourish and persist. Consequently, we examine if and how talkability (item) and initiator position (network) interact to mitigate the negative effects of increasing decay on both Talk $_{k t}$ and Excited $_{k t}$.

Talkability $\times$ Decay. Results reveal significant decay $\times$ talkability interactions for both dependent variables ( $p$ s $<.001$; see Figure 4), indicating that decay has a weaker negative effect on Talk $_{k t}$ and Excited Et $_{t}$ at higher levels of talkability. This shows that 
talkability not only keeps enthusiasm high by directly opposing decay (as indicated by talkability's main effects), but also works indirectly by moderating the effect of decay on collective enthusiasm and the likelihood the item is discussed. In other words, when talkability is high, outcomes are less sensitive to decay.

\section{[INSERT FIGURE 4 ABOUT HERE]}

Talkability mitigates decay by making WOM transmissions more likely or frequent. This can be seen by looking at another variable, $S w i t c h_{k t}$ : the proportion of consumers who did not talk about the item in period $t$-1 but switch to talking about it in period $t$. Talkability has a positive main effect on $\operatorname{Switch}_{k t}(p<.001)$, meaning that higher talkability leads people to be more likely to start talking about the item. There is also a decay $\times$ talkability interaction effect on Switch $_{k t}(p<.001)$ indicating that the effect of decay on weakening enthusiasm and driving people to talk about something other then the focal item is reduced under higher levels of talkability. Because transmissions are more likely and frequent, enthusiasm is reinforced more often and therefore stays higher.

By increasing WOM frequency, higher talkability also increases the average dose size in WOM transmissions, measured by the variable $D_{o s} e_{k t}$ t the mean enthusiasm dose size across all transmitters for simulation run $k$ who transmitted some enthusiasm in period $t$. We regressed Dose $_{k t}$ on the same regressors used in the previous models. There is no main effect of talkability on average dose size ( $p=.27)$, but there is a significant interaction between decay and talkability $(p<.001)$ such that higher talkability diminishes the strength of the negative decay effect on dose size. More talkable items are less sensitive to decay reducing their dose sizes. This is a by-product of talkability’s positive effect on transmission frequency: since more talkable items are talked about 
more often, enthusiasm losses due to decay can be replenished more frequently, which makes high talkability items less sensitive to decay reducing dose sizes.

Initiator Position $\times$ Decay. There is also a significant decay $\times$ initiator position interaction $\left(p<.001\right.$; see Figure 5), such that the negative effect of decay on Excited $_{k t}$ is weaker when close initiators are used to start the contagion process. Similar to what we found for talkability, having close initiators makes the system less sensitive to decay. However, whereas talkability also directly combats decay (i.e., main effects of opposite signs), initiator position's contribution lies solely in moderating the decay effect.

\section{[INSERT FIGURE 5 ABOUT HERE]}

Close initiators mitigate decay by boosting average dose size (rather than frequency). Specifically, initiator position directly affects enthusiasm by leading people

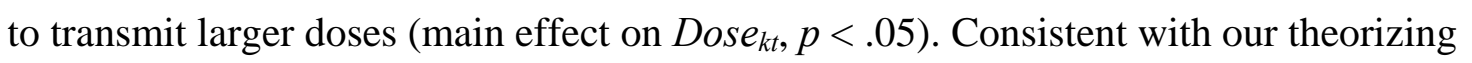
about flows of enthusiasm back-and-forth between initiators and everyone else in the network, compared to peripheral initiators, close initiators on average have shorter path lengths between them and others. Accordingly, the cycles from initiators to non-initiators and back again are also comparatively short. Consequently, close initiators allow average dose sizes to remain higher because enthusiasm travels shorter distances. This means that the doses of enthusiasm that get transmitted will be larger, as this effect indicates. Further, this finding illustrates the importance of multidirectional flows of enthusiasm.

This can also be seen by looking at what happens when high degree initiators (i.e., many social connections) are used. We added a high degree (connectivity) condition to the initiator position factor. We found that high degree initiators (i.e., "hubs" in past research on diffusion) have a similar effect on dose size as peripheral initiators (contrast 
$p>$.05); i.e., a significantly weaker effect than close initiators (contrast $p<.02$ ). Like peripheral initiators, high degree initiators do little to mitigate the negative effects of decay on enthusiasm. This is consistent with our argument about why closeness, rather than degree, is important for persistent epidemics. (Note that despite a positive correlation in real networks between nodes' closeness and degree centralities, the topranked nodes on closeness and degree used here as initiator groups had little overlap.)

Summary. Taken together, these interactions demonstrate how item and network characteristics can mitigate the effect of decay on collective enthusiasm and epidemic persistence. Importantly, the results illustrate multiple routes to enthusiasm reinforcement — either by changing transmission frequency or dose size. Consistent with our perspective, the network position of initiators works through affecting dose size by changing how far enthusiasm must flow between consumers over the network. An item's talkability, on the other hand, drives collective enthusiasm though its affect on transmission frequency (which also has the by-product of boosting average dose size).

\section{Interchangeable Effects?}

We also examined whether the decay-mitigating effects of high talkability and close initiators are interchangeable through a three-way interaction of decay, talkability, and initiator position. This interaction was significant $(p<.001)$. When close initiators are used, the decay $\times$ talkability interaction is smaller; and, conversely, when talkability is higher, the decay $\times$ position interaction is smaller (such that at higher levels of talkability close and peripheral initiators have similar effects). This indicates a reasonable degree of interchangeability between having high talkability items versus relying on close 
initiators. Either talkability or close initiators can be useful in helping to combat decay, but there does not appear to be an additional benefit of having both present. This indicates that both enthusiasm reinforcement mechanisms (increased frequency, increased dose size) can be independently effective in weakening the effect of decay and thus creating more persistent epidemics.

\section{GENERAL DISCUSSION}

The current work integrates psychological and sociological perspectives to increase understanding of social epidemics. In particular, we investigate how word-ofmouth between consumers spreads over social networks and helps sustain social epidemics for particular cultural items. We developed a micro individual-level model of social transmission behaviors based on psychologically plausible assumptions and applied it to a social network to see, though an extensive simulation-based study, how item and network characteristics drive persistent collective enthusiasm. Given that enthusiasm and interest naturally decay over time, we examined how WOM transmitted over social network ties can reinforce consumers' enthusiasm for cultural items, thereby combating decay and bolstering epidemic persistence. By bridging micro-level transmission processes and macro-level diffusion outcomes we not only shed light on the outcomes themselves, but also the processes that drive them.

Our findings show that network and item characteristics can jointly encourage WOM, which in turn, reinforces enthusiasm and bolsters collective enthusiasm. While prior work has tended to consider item- or network-related aspects in isolation, we find 
that these factors can moderate each other's effects and therefore work in combination to drive persistent social epidemics. This means that epidemics may occur even in situations where they might not have been predicted by either individual factor alone.

Further, these results shed light on the underlying mechanisms behind these collective phenomena. Both item talkability and initiator position mitigate the negative effects of decay (which is also an item characteristic) and make the overall system less sensitive to natural declines in enthusiasm. However, they tend to do this in different ways. Talkability reinforces enthusiasm by increasing the likelihood that people will talk about the item (which in turn keeps dose sizes larger). Regardless of how enthusiastically people talk (i.e., dose size), more frequent conversations provide more frequent doses of enthusiasm, which keeps enthusiasm high (e.g., this is analogous to a "pulsing” advertising campaign). Using close initiators reinforces enthusiasm by helping WOM spread over larger network distances more quickly, which maintains higher average doses of enthusiasm. Importantly, it is closeness (based on distances) and not degree (based on direct connections) that matters. Our ancillary analyses illustrate that high degree initiators were no better than peripheral initiators when it came to affecting dose sizes and the enthusiasm reinforcement process.

\section{Directions for Future Research}

Follow up work might more closely examine what drives ongoing enthusiasm and consumption. Product adoption describes an early stage in the consumer-product relationship, but many more stages ensue. Consumers may remain enthusiastic or get bored, keep consuming or stop, and eventually switch to a competitor or abandon the 
product for other reasons (e.g., Berger and Heath 2008). Considering drivers of ongoing enthusiasm and epidemic persistence not only sheds light on diffusion, but also on product lifespans more generally (e.g., why once-popular things die out).

Diffusion research would also benefit from considering a wider array of domains. Existing theorizing has been heavily based on the types of products the literature has tended to investigate. Adopting things that are expensive (e.g., hybrid corn) or risky (e.g. a new drug) requires being relatively certain of the benefits, and thus seeking information from peers to mitigate risk is seen as an important factor influencing whether social contagion will operate (Iyengar et al. 2010). For a host of other products, however, the question is less about risk mitigation and more about whether people are (and remain) enthusiastic. There is little cost involved in trying a website or listening to a new band, but given the multitude of options available, consumers must remember someone told them about a particular option and be enthusiastic enough to give it a try. Further, while the continued usage of hybrid corn or a new drug will depend mostly on product efficacy, in many other instances, continued product enthusiasm depends less on the product itself and more on conversations with others. Part of the utility of listening to a particular musician is talking about them with your friends, and in these instances, continued consumption will depend on the product continuing to be discussed. This is particularly likely in cases where utility is driven by social factors rather than just functional aspects (e.g. using a website or being a fan). Consequently, factors that shape what people talk about, and enthusiasm with which they talk, should drive ongoing consumption and the size and persistence of social epidemics. 
More broadly, while social contagion, WOM, and social networks have recently become popular topics in the marketing science literature, they deserve greater attention in consumer behavior. Quantitative work has convincingly shown that social networks, WOM, and social contagion influence consumer behavior and sales (e.g., Godes and Mayzlin 2004, 2009; Goldenberg et al. 2001, 2004, 2009; Iyengar et al. 2010; Stephen and Toubia 2010), but less in known about the underlying behavioral processes behind these outcomes. Researchers must now shoulder the difficult task of moving beyond these first generation questions to investigate the psychological and sociological aspects underlying the transmission process. Why do people talk about certain products more than others? How do characteristics of conversation partners determine what is discussed? And what are the dynamics of conversations between people over time? These are only a handful of the many important questions that remain unanswered.

In closing, social epidemics are complex phenomena. Individuals decide what to talk about based on psychological processes and item characteristics, and these discussions are situated in a broader social network structure. Beginning to understand these multiply determined outcomes requires not only digging deeply within disciplinary traditions, but also linking more effectively across them. Insights on memory, emotion, social influence, identity, social networks, and more must be brought together more cohesively before integrative progress is made. Only then will researchers and practitioners truly understand what drives social epidemics. 


\section{APPENDIX}

\section{A. Details for Individual-Level Model of Social Transmission Behaviors}

Decay rate parameter $\left(\delta_{i}\right)$. Consumers' decay rates are distributed around an item-specific decay rate. Specifically, $\delta_{i} \sim t N\left(\delta\right.$, $\left.\xi^{2}\right)$; i.e., from a truncated normal distribution (between 0 and 1 , since the decay rate parameter lies in this range). The mean is $\delta$, which is the item-specific decay rate. The consumer-level heterogeneity corresponding to the fact that some people hold interest longer than others is introduced through the variance around this mean. In the simulation study we used this truncated normal distribution with standard deviation $=.1$. This level of variance was large enough to induce sufficient heterogeneity in decay rates while ensuring that most of the influence of decay was item-specific and did not vary too widely across consumers. A central-mass distribution such as the normal or truncated normal is therefore appropriate given that we wanted most of the weight of decay to be on the item-specific part.

Enthusiasm mixture weight parameter $\left(\gamma_{j}\right)$. This parameter, $\gamma_{j} \in[0,1]$, is the self-versus-other enthusiasm weight that is specific to the transmitter $(j)$. Those transmitters who are more self-focused (other-focused) will have a higher (lower) value for $\gamma_{j}$. We assume for all $N$ consumers in the population $\gamma_{j} \sim \operatorname{Uniform}(0,1)$.

Actor- versus item-specific component weight parameter $\left(\alpha_{j}\right)$. This

parameter, $\alpha_{j} \in[0,1]$, is the weight transmitters $(j)$ put on the actor-specific part of the transmission probability (based on theirs and their receiver's enthusiasm) versus the itemspecific part (item characteristic of talkability). Those transmitters who are more actor enthusiasm-focused (item talkability-focused) will have a higher (lower) value for $\alpha_{j}$. We 
assume for all $N$ consumers in the population $\alpha_{j} \sim \operatorname{Uniform}(0,1)$. Note that this parameter is included in equation 2 because heterogeneity across consumers (as transmitters) is important for the sake of model realism. The alternative would be to assume a way that these components of the transmission probability are mixed (e.g., taking the arithmetic mean). While our distributional assumption implies that, in expectation, this will be the case because $E\left(\alpha_{j}\right)=.5$ (which is a fair base assumption), in the simulation study this will obviously not always be the case and thus, practically, there will be heterogeneity in how consumers-as-transmitters mix these two components of $p_{i j t}$.

\section{B. Details for Initiator Enthusiasm}

Initiators start with enthusiasm above a positive threshold, defined by the parameter $\phi$. Initiators' enthusiasm levels are specified as $Y_{i, t=1}^{\text {initator }} \sim \operatorname{Uniform}(\phi, 1)$. In other words, in our simulation study, the enthusiasm levels of the $N_{\text {initiators }}$ will be uniformly distributed been the threshold and the maximum possible value of 1 . This is done to allow for heterogeneity in initiator-consumers’ responses to marketing actions that establish their nonzero starting levels of enthusiasm (e.g., messages sent in a viral campaign targeting these consumers as "seeds"). This parameter is not of primary importance but we allow it to vary to show that our results are robust to variation in this threshold. This parameter, as a covariate in the statistical analysis, did not interact with anything.

\section{Details on Network Centrality and Closeness}

Closeness centrality is inversely proportional to the average degrees of separation one is from others (mean geodesic distance, or average shortest path length). Note that 
while degree and closeness are often correlated in real social networks (making it hard to identify whether it is degree or closeness driving outcomes), it is possible to have low correlations between degree and closeness centralities between groups of initiators. For instance, the $k_{1}$ people with the highest degree and the $k_{2}$ people with the highest closeness in a social network do not necessarily completely or even majorly overlap, provided that $k_{1}$ and $k_{2}$ are small enough relative to the number of nodes in the network.

In the network used in our simulation study there was a reasonable correlation between degree and closeness (.56). The rank-order Spearman correlation, however, was small (.03). We designated two groups of 20 people each as initiators (out of 1,000 people in the entire network). The groups of the top-20 degree and the top-20 closeness people only had seven overlapping people. This reduces the possibility that any effect of initiator position (based on closeness) is strongly confounded by initiators' degrees. Underscoring the difference in these measures, our results differed substantially when high degree versus high closeness individuals were used in the analysis.

\section{Details on the Social Network Used in the Simulation Study}

The network had $N=1,000$ nodes (consumers) and was connected. This means that every other node could be reached in a finite number of steps from each node in the network. While in large real-world social networks people cannot always reach everyone else in a finite number of steps, larger networks can be decomposed into smaller subnetworks that are connected (also referred to as connected components). Hence, the network mimics a connected component or sub-network of a larger-scale network. 
We used a network generation algorithm to create the static network structure used in our simulation study. The algorithm was based on Newman and Park (2003). We generated a realistic social network structure where people belong to "communities" and can only be connected to a person if they are in at least one of the same communities. Since social networks are different from many other types of networks, random graphs are not ideal. Our population has $N=1,000$ consumers. The underlying community affiliation structure has $N_{C}=25$ communities that people can belong to, with each having a capacity of $M_{c}$ people (for $c=1, \ldots, N_{\mathrm{C}}$ ), with $M_{c} \sim \operatorname{Poisson}\left(N / N_{C}\right)$. Person $i$ is a member of $h_{i}$ communities, where $h_{i} \sim$ Poisson(2). Specifically, if two people are members of the same community then they have a $2 \%$ probability of being connected in the social network. This $2 \%$ probability ensured that the network was sparse and had properties commonly found in real social networks: e.g., mean geodesic distance of approximately 4, mean clustering coefficient of approximately .03, and a skewed and approximately power-law degree distribution. Other realistic structures were tested and the results we found were robust to variations in structure (while still keeping it realistic).

\section{E. Details on Beta Regression Model}

Since Talk $_{k t}$ Excited $d_{k t}$, and Switch $_{k t}$ are proportions (i.e., values lie between 0 and 1), a generalized linear model was used to model these dependent variables as conditionally beta-distributed (conditional on the regressors). The proper distributional assumption—in this case beta, for proportions—reduces potential estimation bias. See Smithson and Verkuilen (2006) for an overview of beta regression. 


\section{REFERENCES}

Bass, Frank M. (1969), “A New Product Growth Model for Consumer Durables,” Management Science, 15 (5), 215-227.

Berger, Jonah and Chip Heath (2008), "Who Drives Divergence? Identity-Signaling, Outgroup Dissimilarity, and the Abandonment of Cultural Tastes," Journal of Personality and Social Psychology, 95 (3), 593-607.

_ and Katherine Milkman (2010), "What Spreads? The Talkability of News," working paper, University of Pennsylvania.

Castellano, Claudio, Santo Fortunato, and Vittorio Loreto (2009), "Statistical Physics of Social Dynamics," Review of Modern Physics, 81, 591.

Cheema, Amar and Andrew M. Kaikati (2010), "The Effect of Need for Uniqueness on Word of Mouth," Journal of Marketing Research, 47 (3).

Coleman, James, Elihu Katz, and Herbert Menzel (1957), "The Diffusion of an Innovation Among Physicians,” Sociometry, 20 (4), 253-270.

Dodds, Peter Sheridan and Duncan J. Watts (2004), “A Generalized Model of Social and Biological Contagion,” Journal of Theoretical Biology, 232 (2005), 587-604.

Fast, Nathanael J., Chip Heath and George Wu (2009), “Common Ground and Cultural Prominence: How Conversation Strengthens Culture,” Psychological Science, 20, 904-911.

Frenzen, Jonathan and Kent Nakamoto (1993), "Structure, Cooperation and the Flow of Market Information,” Journal of Consumer Research, 20 (December), 360-375.

Godes, David and Dina Mayzlin (2004), "Using Online Conversations to Study Word-ofMouth Communication,” Marketing Science, 23 (4), 545-560.

— and — (2009), "Firm-Created Word-of-Mouth Communication: Evidence from a Field Test,” Marketing Science, 28 (4), 721-739.

Goldenberg, Jacob, Barak Libai, and Eitan Muller (2001), "Talk of the Network: A Complex Systems Look at the Underlying Process of Word-of-Mouth,” Marketing Letters, 12 (3), 211-223.

— — _ - and —— (2004), "Complex, Yet Simple: Cellular Automata as an Enabling Technology in Marketing Strategy Research.” In Christine Moorman and Donald R. Lehmann (eds), Assessing Marketing Strategy Performance, Cambridge, MA: Marketing Science Institute.

—_ - Sarit Moldovan and Eitan Muller (2007) "The NPV of Bad News," International Journal of Research in Marketing, 24, pp.186-200.

— - Sangman Han, Donald R. Lehmann, and Jae Weon Hong (2009), “The Role of Hubs in the Adoption Process,” Journal of Marketing, 73 (2), 1-13. 
Golder, Peter N. and Gerard J. Tellis (2004), “Going, Going, Gone: Cascades, Diffusion, and Turning Points of the Product Life Cycle,” Marketing Science, 23 (2), 207-218.

Greene, William H. (2003), Econometric Analysis, $5^{\text {th }}$ ed., New York: Prentice Hall.

Grice, H. Paul. (1975), “Logic and Conversation.” In P. Cole \& J. L. Morgan (Eds.), Syntax and Semantics 3: Speech Acts, New York: Academic Press.

Heath, Chip, Chris Bell and Emily Sternberg (2001), "Emotional Selection in Memes: The Case of Urban Legends,” Journal of Personality and Social Psychology, 81 (6), 1028-1041.

Hirschman, Elizabeth C. (1980), "Innovativeness, Novelty Seeking, and Consumer Creativity,” Journal of Consumer Research, 7 (3), 283-295.

Iyengar, Raghuram, Christophe Van den Bulte and Thomas W. Valente (2010), “Opinion Leadership and Social Contagion in New Product Diffusion,” Marketing Science, forthcoming.

Kashima, Yoshihasa (2008), “A social psychology of cultural dynamics: examining how cultures are formed, maintained, and transformed,” Social and Personality

Psychology Compass, 2, 107-120.

Leskovic, Jure, Lada Adamic, and Bernardo A. Huberman (2007), “The Dynamics of Viral Marketing," ACM Transactions on the Web, 1 (1).

Libai, Barak, Ruth Bolton, Marnix Bügel, Ko DeRuyter, Oliver Götz, Hans Risselada, and Andrew T. Stephen (2010), "Customer to Customer Interactions: New Opportunities and Research Directions,” Journal of Service Research, forthcoming.

—_, Eitan Muller and Renana Peres (2005), “The Role of Seeding in Multi-Market Entry,” International Journal of Research in Marketing, 22 (December) 375-393.

— _ - and (2009), "The Effect of Within-Brand and Cross-Brand Communication on Competitive Growth,” Journal of Marketing, 73 (1), 19-34.

Lusch, Robert F. and Nicholas Tay (2004), “Agent-Based Modeling: Gaining Insight Into Firm and Industry Performance.” In Christine Moorman and Donald R. Lehmann (eds), Assessing Marketing Strategy Performance, Cambridge, MA: Marketing Science Institute.

Mark, Noah (1998), “Birds of a Feather Sing Together,” Social Forces, 77 (2), 453-485.

Moldovan, Sarit, Jacob Goldenberg, and Amitava Chattopadhyay (2006), "What Drives Word-of-Mouth? The Roles of Product Originality and Usefulness,” MSI Report 06-111, Cambridge, MA: Marketing Science Institute.

Newman, Mark E.J. and Juyong Park (2003), "Why Social Networks are Different from Other Types of Networks,” Physical Review E, 68, 036122.

de Nooy, Wouter, Andrej Mrvar, Vladimir Batagelj (2005), Exploratory Social Network Analysis with Pajek, Cambridge, UK: Cambridge University Press.

Rogers, Everett M. (1976), Diffusion of Innovations, New York, NY: Free Press. 
and F. Floyd Shoemaker (1971), Communication of Innovations, New York, NY: Free Press.

Schaller, Mark, Lucian Gideon Conway III and Tracy L. Tanchuk (2002), “Selective Pressures on the Once and Future Contents of Ethnic Stereotypes: Effects of the Communicability of Traits,” Journal of Personality and Social Psychology, 82 (6), 861-877.

Schaller, Mark and Christian S. Crandall (2004), The Psychological Foundations of Culture, Mahwah, NJ: Lawrence Erlbaum Associates.

Smithson, Michael and Jay Verkuilen (2006), “A Better Lemon Squeezer? MaximumLikelihood Regression With Beta-Distributed Dependent Variables,” Psychological Methods, 11 (1), 54-71.

Stephen, Andrew T. and Donald R. Lehmann (2010), "Drivers of Word-of-Mouth Transmission at the Individual Level and Consequences for Diffusion,” working paper, INSEAD.

_ and Olivier Toubia (2010), “Deriving Value from Social Commerce Networks,” Journal of Marketing Research, 47 (2), 215-228.

Toubia, Olivier, Jacob Goldenberg and Rosanna Garcia (2010), “A New Approach to Modeling the Adoption of New Products: Aggregated Diffusion Models,” working paper, Columbia University.

Van den Bulte, Christophe and Gary L. Lilien (2001), “Medical Innovation Revisited: Social Contagion versus Marketing Effort,” American Journal of Sociology, 106 (5), 1409-1435.

Watts, Duncan J. (2002), “A Simple Model of Global Cascades on Random Networks,” Proceedings of the National Academy of Sciences of the United States of America, 99 (9), 5766-5771.

— (2007), “Challenging the Influentials Hypothesis,” WOMMA Measuring Word of Mouth, 3, 207.

— and Peter Sheridan Dodds (2007), "Influentials, Networks, and Public Collective Enthusiasm Formation,” Journal of Consumer Research, 34 (4), 441-458.

Wojnicki, Andrea and David Godes (2008), “Word-of-Mouth as Self-Enhancement,” working paper, University of Toronto.

Wu, Fang and Bernardo A. Huberman (2007), "Novelty and Collective Attention," Proceedings of the National Academy of Sciences of the United States of America, 104 (45), 17599-17601. 
TABLE 1

MANIPULTED PARAMETERS IN SIMULATION STUDY

\begin{tabular}{lll}
\hline Parameter & $\begin{array}{c}\text { Levels Used in } \\
\text { Simulation Study }\end{array}$ & Definition \\
\hline
\end{tabular}

\section{Item Characteristics}

Decay, $\delta \quad 0 ; .025 ; .05 ; .075 ; .1 ; \quad$ The per-period rate at which a consumer's $[0,1] \quad .125 ; .15 ; .175 ; .2 \quad$ enthusiasm for a product decays between periods.

Talkability, $v \quad 0 ; .1 ; .2 ; .3 ; .4 ; .5 ; .6 ; \quad$ The probability that a person talks to a $[0,1] \quad .7 ; .8 ; .9 ; 1 \quad$ friend about the item irrespective of their and their friend's current enthusiasm for that item.

\section{Network Characteristic}

\begin{tabular}{|c|c|c|}
\hline Initiator position & $\begin{array}{l}\text { Close (high } \\
\text { closeness); } \\
\text { Peripheral (low } \\
\text { closeness) }\end{array}$ & $\begin{array}{l}\text { Closeness centrality is a network centrality } \\
\text { measure that is related to the length of the } \\
\text { paths that connect a consumer to other } \\
\text { consumers in a social network. Close (high) } \\
\text { people have a relatively short average path } \\
\text { length between them and others. Peripheral } \\
\text { (low) people have a relatively long average } \\
\text { path length between them and others. }\end{array}$ \\
\hline
\end{tabular}

\footnotetext{
Note: the levels for decay do not cover the full [0,1] parameter space for $\delta$. This was because in pretests it
} was found that above the level of . 2 the contagion process was killed/overwhelmed by decay. 
TABLE 2

PARAMETER ESTIMATES FOR MAIN RESULTS

Parameter Estimates
(Standard Errors)

Excited $_{k t}$

Talk

\section{Main Effects}

Decay

$-25.24(1.108)^{*} \quad-9.42(1.000)^{*}$

Talkability

$\begin{array}{llll}4.72 & (.214)^{*} & .59 & (.201)^{*}\end{array}$

Initiator position $($ base $=$ peripheral $)$

$-.17 \quad(.177)^{\mathrm{ns}}$

$.14(.168)^{\mathrm{ns}}$

\section{Lagged Effects}

Excited $_{t-1}$

$2.62(.031)^{*}$

$\mathrm{n} / \mathrm{a}$

Talk $_{t-1}$

$10.26(.072)^{*} \quad 4.74 \quad(.003)^{*}$

Interaction Effects

Decay $\times$ talkability

$30.40(1.832)^{*} \quad 10.55(1.689)^{*}$

Decay $\times$ position

$7.23(1.544)^{*} \quad .67(1.414)^{\mathrm{ns}}$

Talkability $\times$ position

$.20 \quad(.298)^{\mathrm{ns}}$

$-.17 \quad(.284)^{\mathrm{ns}}$

Decay $\times$ talkability $\times$ position

$-8.66(2.568)^{*} \quad-1.08(2.389)^{\mathrm{ns}}$

\section{Other Effects and Model Parameters}

\begin{tabular}{lrlrl} 
Excited threshold & -1.88 & $(.115)^{*}$ & .04 & $(.109)^{\mathrm{ns}}$ \\
Intercept & -1.85 & $(.138)^{*}$ & -2.45 & $(.131)^{*}$ \\
Random effect variance & .51 & $(.027)^{*}$ & .47 & $(.024)^{*}$ \\
Beta distribution scale parameter & 62.03 & $(.225)^{*}$ & 242.65 & $(.870)^{*}$ \\
\hline
\end{tabular}
${ }^{*} p<.01,{ }^{\text {ns }}$ not significant. 


\section{FIGURE 1}

ILLUSTRATION OF THE EFFECT OF ENTHUSIASM DECAY ON SOCIAL CONTAGION OVER A NETWORK

A: Without Enthusiasm Decay

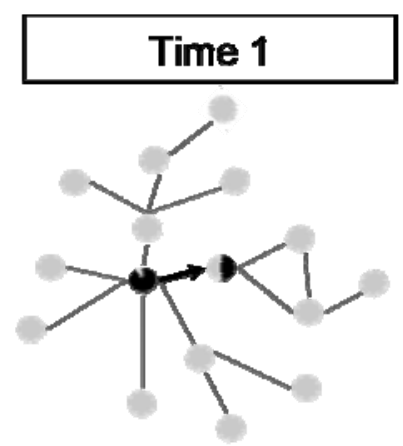

2 out of 16 people with enthusiagm $>0$

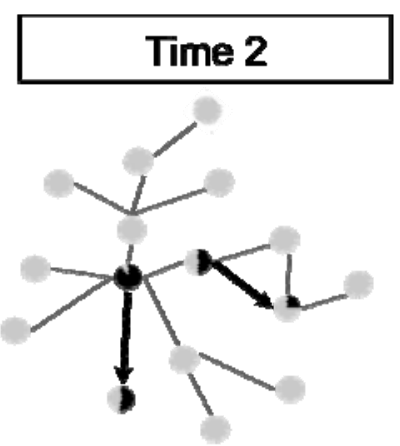

4 out of 16 people with enthusiagm $>0$

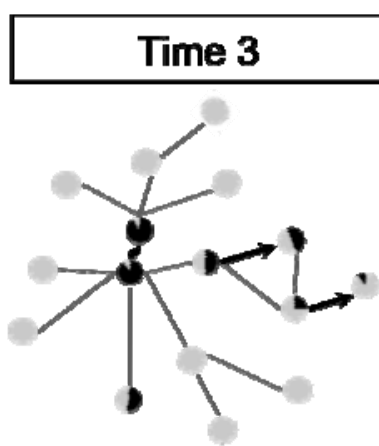

7 out of 16 people with enthusiagm $>0$

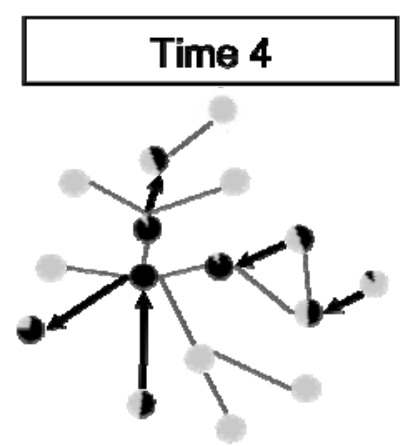

9 out of 16 people with enthusiasm $>0$

\section{B: With Enthusiasm Decay}

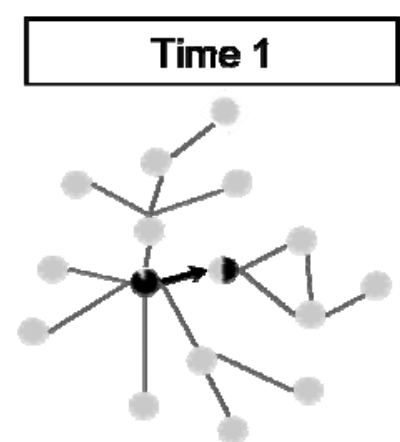

2 out of 16 people with enthusiasm $>0$

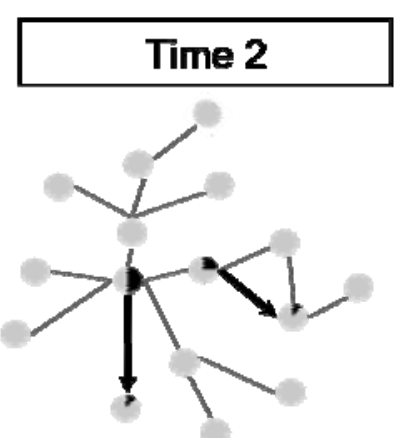

4 out of 16 people with enthusiasm > 0
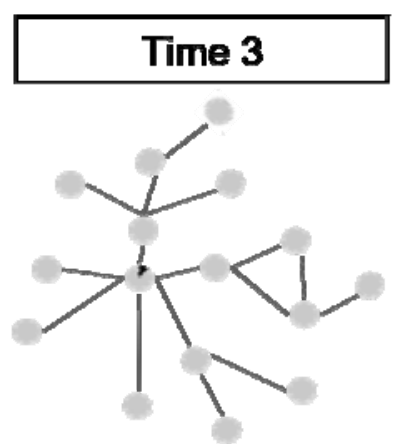

1 out of 16 people with enthusiasm $>0$

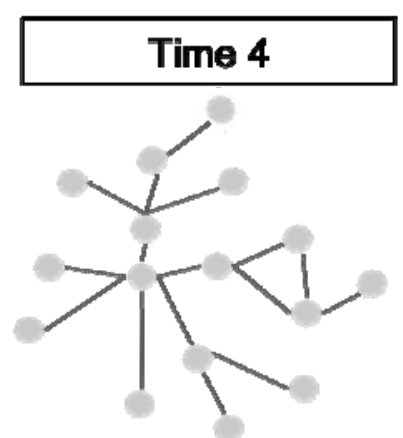

0 out of 16 people with enthusiasm > 0 
FIGURE 2

OVERVIEW OF INDIVIDUAL-LEVEL SOCIAL TRANSMISSION PROCESS

Every period, person $j$ (transmitter) picks one of their network ties (person $i$, receiver) to talk to about something

\section{Start Period $t$}

\section{What is \\ talked about?}

What

is the effect?

Transmitter talks about either the focal item or something else, based on:

1. How enthusiastic they are about the focal item

2. How enthusiastic the person they are talking to (receiver) is about the focal item

3. The focal item's talkability

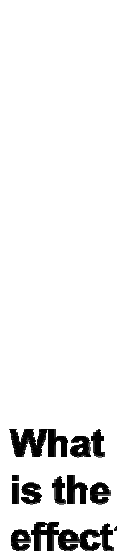

\section{End of Period $t$}

Decay on Transmitter's and Receiver's end-of-period enthusiasm 


\section{FIGURE 3}

ILLUSTRATION OF THE EFFECT OF A CLOSE VERSUS A PERIPHERAL INITIATOR ON ENTHUSIASM

\section{REINFORCEMENT}

A: Close Initiator

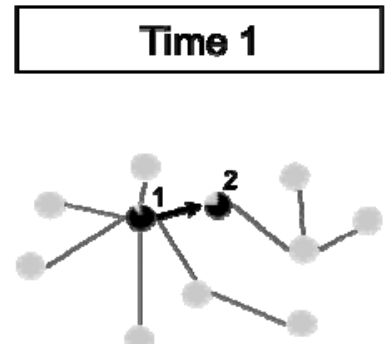

- Inlisator la 1, "cloes"

- 1 trangrits a dose to 2

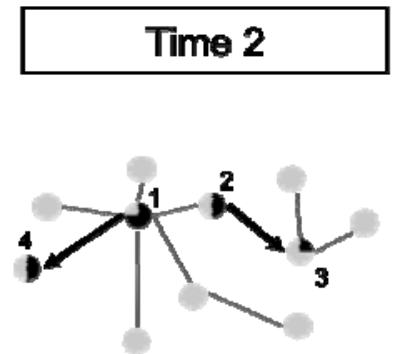

- Enthuedeam docay for 1 and 2

- 1 transmits a dose to 4

- 2 transmits a dose to 3

B: Peripheral Initiator

Time 1
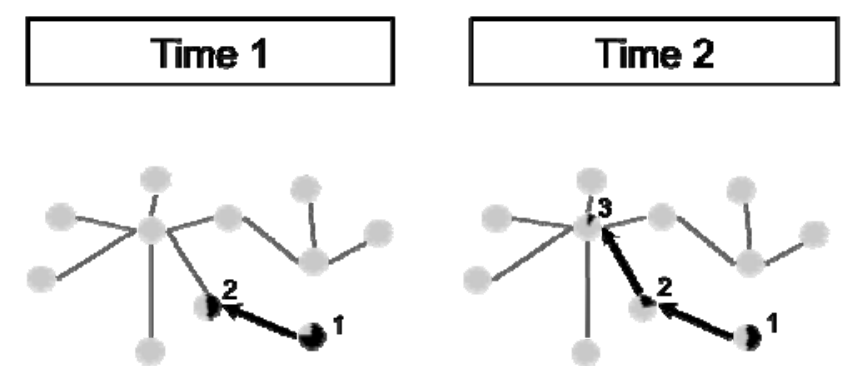

- Intitator b 1, peripherar

- 1 transmits a dose to 2

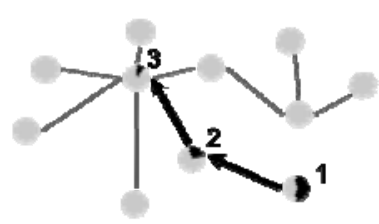

- Enthusiarm docay for 1 and 2

- Enthusiasm docay for 1 and 2 - Enthustaem decay for 1-3

- 2 transmits a doso to 3

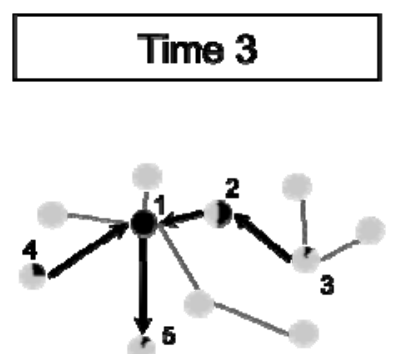

- Enthualaen decay for 1-4

- 1 transmits a dose to 5

- 2 transmits a reinforeing dose to 1

- 3 tranmits a reinforcing does to 2

- 4 transmits a reinforeting dose to 1
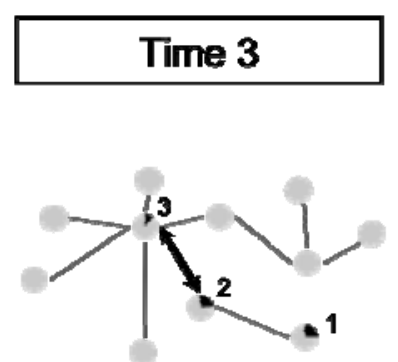

- 2 tranmits a reinforeing dose to 3

- I transmits a reinforcing dose to 1

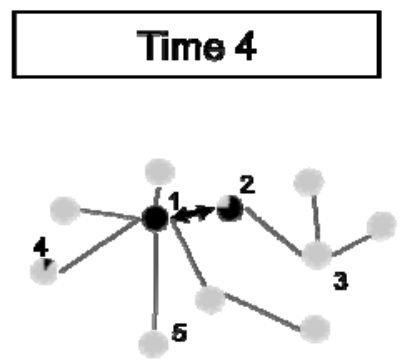

- Enthualeam decay for 1-5

- 1 transite a reinforching dose to 2

- 2 transernits a reinforeing dose to 1
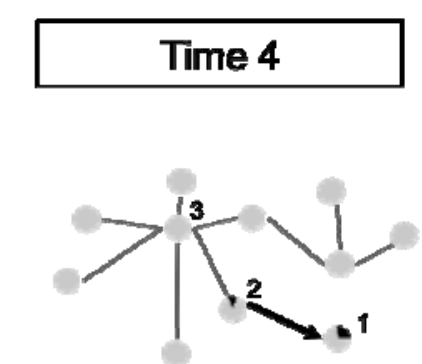

- Enthustaem docay for 1-3

- 1 by chance does not trangmit

- 2 tranamils a reinforeing doee to 1 
FIGURE 4

DECAY $\times$ TALKABILITY INTERACTION EFFECT ON COLLECTIVE ENTHUSIASM

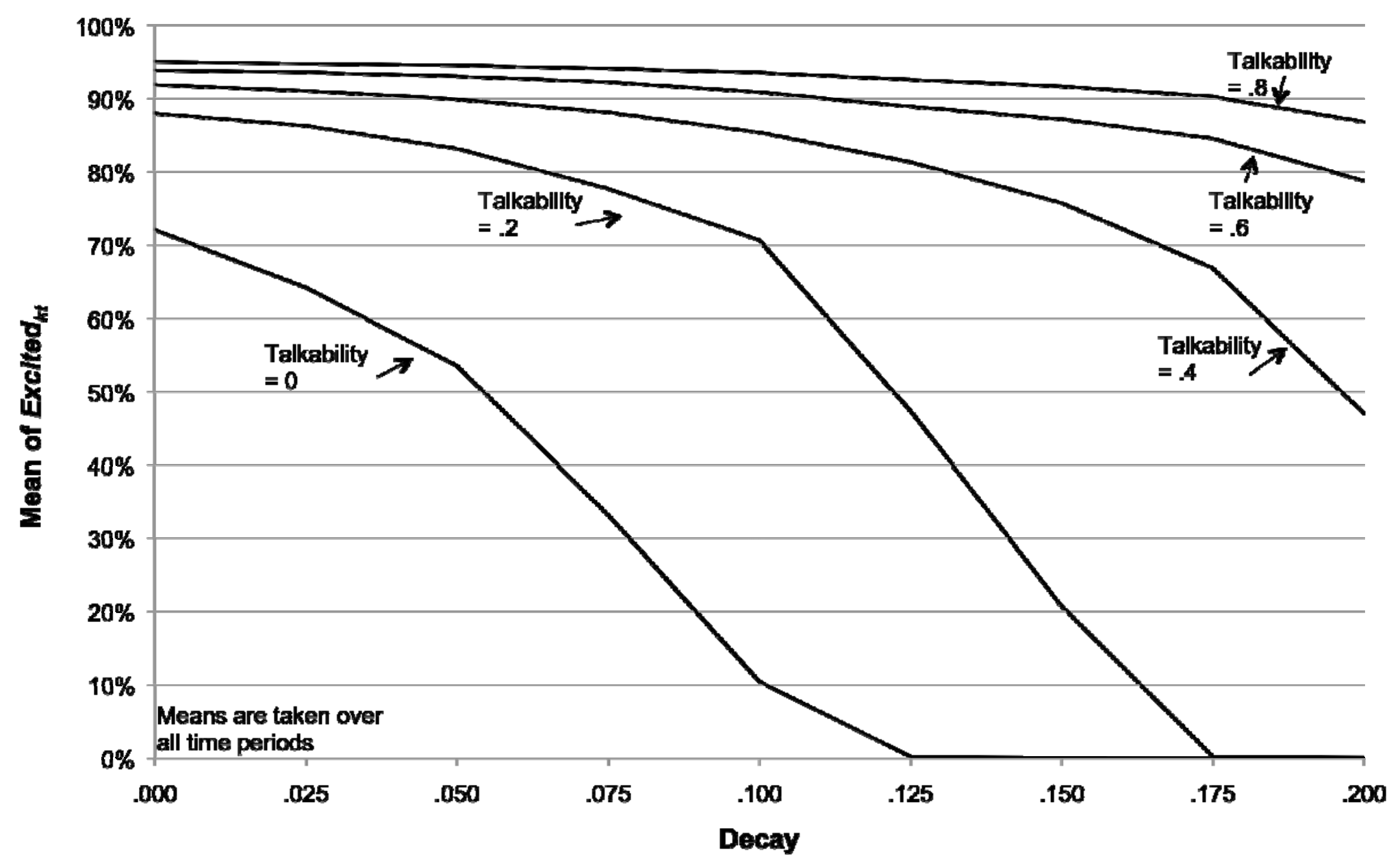


FIGURE 5

DECAY $\times$ INITIATOR POSITION INTERACTION EFFECT ON COLLECTIVE ENTHUSIASM

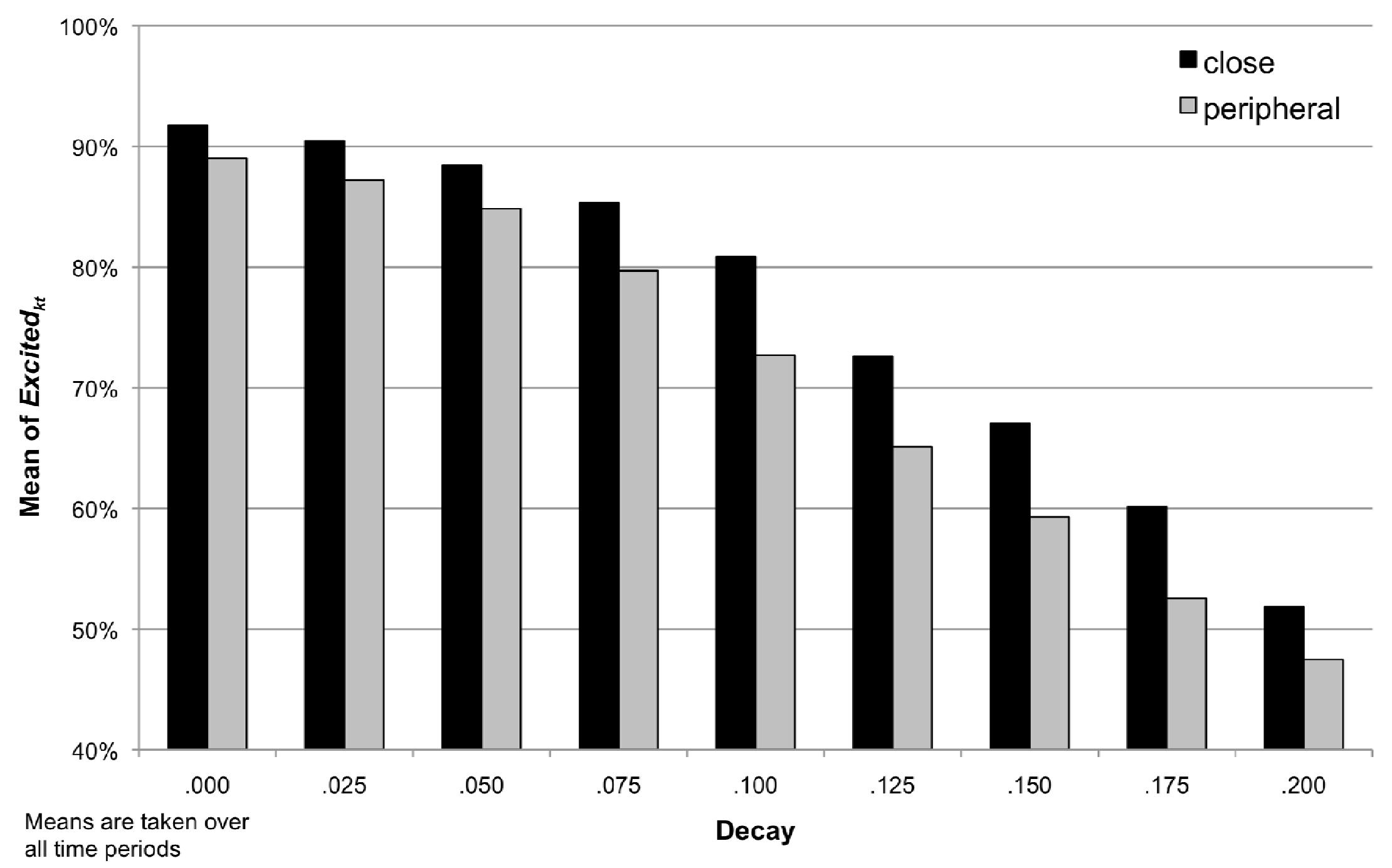

